\title{
FZD2 inhibits the cell growth and migration of salivary adenoid cystic carcinomas
}

\author{
LIN-CAN DING $^{1 *}$, XIAO-YU HUANG ${ }^{1 *}$, FEI-FEI ZHENG ${ }^{1 *}$, JIAN XIE $^{1}$, LIN SHE $^{1}$, \\ YAN FENG ${ }^{1}$, BO-HUA SU ${ }^{1}$, DA-LI ZHENG ${ }^{2}$ and YOU-GUANG LU ${ }^{1}$ \\ ${ }^{1}$ Department of Preventive Dentistry, Affiliated Stomatological Hospital, Fujian Medical University, Fuzhou 350002; \\ ${ }^{2}$ Key Laboratory of Ministry of Education for Gastrointestinal Cancer, School of Basic Medical Sciences, \\ Fujian Medical University, Fuzhou 350108, P.R. China
}

Received November 14, 2014; Accepted October 24, 2015

DOI: $10.3892 / o r .2015 .3811$

\begin{abstract}
Several studies have reported that FZD2 regulates tumor biology in a complex manner. The aim of the present study was to identify the role of FZD2 in the cell growth and metastasis of salivary adenoid cystic carcinomas (SACCs). The expression of FZD2 in ACC-83 and ACC-LM cells were measured with real-time PCR. Immunohistochemical staining was used to detect the expression of FZD2 in clinical SACC samples with or without metastasis. Cell proliferation and Transwell assays were performed to explore the effects of FZD2 on cell growth and migration following the silencing of FZD2 with small interference RNAs and the overexpression of FZD2 with plasmid. Our data showed that FZD2 was downregulated in ACC-LM cells, which are an adenoid cystic carcinoma cell line with high metastatic potential, compared to ACC-83 cells, which have low metastatic potential. Additionally, the expression of FZD2 was lower in SACC tissues with metastasis compared to SACC tissues without metastasis $(\mathrm{P}<0.05)$. Cell proliferation and migration of ACC- 83 cells were increased after the knockdown of FZD2 and decreased following overexpression of FZD2. Knockdown of FZD2 downregulated the expression of PAI-1. Our results suggest that FZD2 may be a tumor suppressor gene in SACCs that inhibits cell growth and migration.
\end{abstract}

Correspondence to: Professor You-Guang Lu, Department of Preventive Dentistry, Hospital of Stomatology, Fujian Medical University, 246 Yangqiao Zhong Road, Fuzhou 350002, P.R. China E-mail: fjlyg63@163.com

Dr Da-Li Zheng, Key Laboratory of Ministry of Education for Gastrointestinal Cancer, School of Basic Medical Sciences, Fujian Medical University, 1 Xueyuan Road, Fuzhou 350108, P.R. China E-mail: dalizheng@mail.fjmu.edu.cn

*Contributed equally

Key words: salivary adenoid cystic carcinoma, FZD2, Wnt signal pathway, cell proliferation, cell migration

\section{Introduction}

Salivary adenoid cystic carcinomas (SACCs) are malignant tumors of the head and neck and are characterized by unique clinical features and behaviors. SACCs occur in the major and minor salivary glands and disperse to the oral and oropharyngeal mucosa, tracheobronchial tree and the esophagus. The biological properties of this dispersal include slow and indolent growth, a low probability of regional nodal metastasis, a high propensity for perineural migration and distant metastasis, and a high incidence of recurrence. It has been reported that $40-60 \%$ of SACC patients develop distant metastases in the lungs, bone and soft tissues (1-3). Distant metastases lead to poor patient survival. Therefore, the search for molecules that are relevant to SACC migration and metastasis and the study of the corresponding molecular mechanisms will introduce a new experimental foundation and provide possible molecular targets for the early diagnosis, therapy and prognostic analysis of SACC (4).

The Wnt signal represents a path that has been highly versatile in the process of biological evolution (5) and consists of numerous signaling proteins that include extracellular factors (Wnt), transmembrane receptors (frizzled and FZD), a cytoplasmic protein ( $\beta$-catenin), nuclear transcription factors (TCF and LEF) and others. These signaling proteins are primarily activated in the embryonic development process and play significant roles in embryo development and tissues differentiation. Increasing numbers of studies have indicated that tumorigenesis and tumor metastasis are related to the abnormal activation of the Wnt signaling pathway (6-8). The FZD gene family contains the genes for the receptors of the Wnt signaling pathway, and the receptor proteins (frizzled proteins) encoded by FZD can combine with Wnt ligands to activate the Wnt signaling pathway (9-11). It has been reported that FZD accelerates the occurrence and development of carcinomas $(9,12,13)$, but FZD has also been reported to be tumor suppressor gene in other studies $(14,15)$. At present, the roles of FZD2 in SACC remain unclear.

In this study, we aimed to explore the differences in the expression of FZD2 between ACC-83 and ACC-LM cells using quantitative real-time PCR. We also analyzed the expression of FZD2 in clinical SACC samples with and without metas- 
tasis using immunohistochemistry to examine the correlation between FZD2 expression and SACC metastasis. We silenced FZD2 via small interfering RNA (siRNA) and overexpressed FZD2 using plasmid to elucidate the effects of FZD2 on SACC growth and migration. We explored the expression of E-cadherin, vimentin, MET and PAI-1 after FZD2 was reduced in SACC cells.

\section{Materials and methods}

Cell culture and clinical samples. The highly metastatic cell line SACC-83 and the minimally metastatic cell line SACC-LM were provided by the Peking University School of Stomatology. The cells were maintained in 1640 with $10 \%$ fetal bovine serum (FBS) (both from Gibco, USA), and incubated in a humidified atmosphere of $95 \%$ air and $5 \% \mathrm{CO}_{2}$ at $37^{\circ} \mathrm{C}$. Pathologically diagnosed tissue samples were obtained from the First Affiliated Hospital of Fujian Medical University and were collected between 1997 and 2008. Forty paraffinembedded samples, including 19 cases with metastasis or recurrence and 21 cases without metastasis or recurrence, had been kept on-file by the Pathology Department. This study was approved by the Institutional Review Board of Fujian Medical University, and written informed consent was obtained from each participant.

Quantitative real-time PCR analysis. Total RNA was extracted from the SACC cells using TRIzol reagent (\#15596018; Invitrogen, Carlsbad, CA, USA), and RNA purities and concentrations were detected by ultraviolet spectrometry. The RNAs were separately diluted to the same concentration after being measured and then reverse-transcribed into cDNA with the PrimeScript RT reagent kit (\#RR037A; Takara, Japan). PCR was performed in triplicate using the primers listed in Table I and SYBR Premix Ex Taq ${ }^{\mathrm{TM}}$ (\#RR420A; Takara) according to the manufacturer's instructions. The fluorescence values from the 12 cycles were used as the background signal, and the threshold value was set at 10 times the standard deviation of the fluorescence signals of cycles 4-12. The expression levels were normalized to $\beta$-actin mRNA levels for each sample obtained from parallel assays and analyzed according to Livak and Schmittgen (16).

Immunohistochemical staining assay. The immunohistochemical SP three-step approach was used to stain and analyze the SACC pathological tissues using ovarian carcinomas as a positive control group, and phosphate-buffered saline (PBS) was used in place of the primary antibodies in the negative control groups. After deparaffinization in xylene, the sections were rehydrated in a decreasing gradient of ethanol and washed for $10 \mathrm{~min}$ in PBS ( $\mathrm{pH}$ 7.2). Endogenous peroxidase activity was inhibited by incubation in methanol containing $3 \% \mathrm{H}_{2} \mathrm{O}_{2}$ for $10 \mathrm{~min}$. After several washes in PBS, the sections were blocked with a universal blocking reagent (Maxin, USA) for $10 \mathrm{~min}$ at room temperature and then incubated with the primary antibody against FZD2 (1:5,000 dilution; GR76263-10; Abcam Cambridge, UK) for $1 \mathrm{~h}$ at room temperature. After several washes in PBS, the sections were incubated with a biotin-conjugated secondary antibody (Maxin) for $10 \mathrm{~min}$ at room temperature. After several washes in PBS, the
Table I. Sequences of the primers used in this study.

\begin{tabular}{lll}
\hline Gene & Access no. & \multicolumn{1}{c}{ Sequence } \\
\hline FZD2-F & NM001466 & AGTTCTATCCGCTGGTGAAGGT \\
FZD2-R & NM001466 & GCCCAGAAACTTGTAGCTGAGA \\
ACTB-F & NM001101 & CCTGGCACCCAGCACAAT \\
ACTB-R & NM001101 & GGGCCGGACTCGTCATACT \\
Vimentin-F & NM001292 & TGGACCAGCTAACCAACGACAA \\
Vimentin-R & NM001292 & GTTCAAGGTCAAGACGTGCCAG \\
MET-F & NM001127 & GCTGACTTCTCCACTGGTTCCT \\
MET-R & NM001127 & ACCAAGGTAAACAGGAGCACGA \\
PAI-1-F & NM000602 & GCCAAGAGCGCTGTCAAGAAG \\
PAI-1-R & NM000602 & TTCACCAAAGACAAGGGCCAGG \\
E-cadherin-F & NM004360 & GCTTCCCTCTTTCATCTCCTGA \\
E-cadherin-R NM004360 & GCCACATTTTCTTCTTGCTCCT \\
\hline
\end{tabular}

sections were incubated with streptavidin-peroxidase (Maxin) for $10 \mathrm{~min}$ at room temperature. The sections were rinsed with PBS, and the antibody complexes were visualized by incubation with diaminobenzidine tetrahydrochloride (DAB) chromogen (Maxin). The sections were then counterstained with hematoxylin (Dako, Denmark), dehydrated and examined by light microscopy. All slides were reviewed independently by two pathologists who were blinded to each other's readings. The staining results were assessed on the following three-tier scale: negative indicated no staining, 1+ indicated weak staining and $2+$ indicated strong staining. The immunohistochemical results were graded of 1 of 3 different scores as follows: negative indicated no staining or $1+$ staining in $\leq 30 \%$ of the cells, positive indicated $1+$ staining in $>30 \%$ of the cells or $2+$ staining in $<50 \%$ of cells, and strong positive indicated $2+$ staining in $>50 \%$ of the cells (17).

RNAi and plasmid transfection. Twenty-four hours before transfection, SACC-83 cells in the exponential phase of growth were digested, counted and plated into 6-well plates at $3 \times 10^{5}$ cells/well. The cells were then transfected with siRNAs (18-20) (GenePharma, Shanghai, China) (the sequences are indicated in Table II) using Lipofectamine RNAiMAX (1044526) or plasmid (a kind gift from Changgong Li, Department of Pediatrics, University of Southern California) using Lipofectamine 3000 (1713234) (both from Invitrogen) according to the manufacturer's instructions.

Western blotting. Total cell proteins were extracted, and protein assays were examined with a BCA kit and an ELISA reader. The total proteins were separated by $8 \%$ SDS-PAGE and transferred onto PVDF membranes (Amersham, USA). Subsequently, the membranes were immunoblotted with primary antibodies against FZD2 (1:5,000 dilution; GR76263-10; Abcam) or $\beta$-actin (1:2,000; 1014F; CWBio, Beijing, China) in 5\% bovine serum albumin overnight, washed three times with tris-buffered saline with $0.1 \%$ Tween-20, and incubated with secondary antibody (1:2,000 dilution; Abcam). The immunoreactive protein bands were 
Table II. The sequences of siRNAs used in this study.

\begin{tabular}{lc}
\hline siRNA & \multicolumn{1}{c}{ Sequence (5'-3') } \\
\hline FZD2-605 & 5'-GCGAAGCCCUCAUGAACAATT-3' \\
& 5'-UUGUUCAUGAGGGCUUCGCTT-3' \\
FZD2-931 & 5'-CCCGAUGGUUCCAUGUUCUTT-3' \\
& 5'-AGAACAUGGAACCAUCGGGTT-3' \\
FZD2-1792 & 5'-CCGACUUCACGGUCUACAUTT-3' \\
& 5'-AUGUAGACCGUGAAGUCGGTT-3' \\
Negative control & 5'-UUCUCCGAACGUGUCACGUTT-3' \\
& 5'-ACGUGACACGUUCGGAGAATT-3'
\end{tabular}

visualized via CDP-Star reagent (Roche, USA), and the signals were scanned with a densitometer for semi-quantification of the signal intensities.

Cell viability assay. Cell proliferation was measured by counting the cells in the logarithmic phase with a Cell Counting Kit-8 (\#CK04; Dojindo Kumamoto, Japan). The cells were first transfected with siRNA or plasmid and then plated into a 96-well plate. Cells from each group were plated in 3-wells, and each well had $2 \times 10^{3}$ cells. The absorbance of each well was measured with a microplate reader at the same time over 5 consecutive days. This process was repeated in triplicate for the statistical analyses and to draw the corresponding curves.

Colony formation assay. Twenty-four hours after siRNA or plasmid transfection, the cells were plated into 6-well plates (500 cells/well) and cultured for 2 weeks. Colonies were fixed with cold methanol for $10 \mathrm{~min}$ and stained with $1 \%$ crystal violet for $30 \mathrm{~min}$.

In vitro cell migration assay. The cell migration assays were performed in 24-well Transwell chambers (\#353097; BD Biosciences, USA). Twenty-four hours after siRNA or plasmid transfection, the cells were serum starved for $24 \mathrm{~h}$ and then collected with 1640 with $0.1 \%$ FBS. The cells were plated into the upper chamber at a density of $1.0 \times 10^{5}$ cells/well, and $700 \mu \mathrm{l}$ of 1640 containing $10 \% \mathrm{FBS}$ was added to the lower chamber. Forty-eight hours later, the cells in the upper chamber were removed with cotton swabs and stained with $1 \%$ crystal violet for $10 \mathrm{~min}$. The cells of five random microscopic fields (x200) were counted and photographed.

Statistical analyses. The data were analyzed with the SPSS 22.0 statistics software package. Rank-sum tests were used to compare the rates between the two groups of immunohistochemistry data, and multi-sample average one-way ANOVA tests were used for between group comparisons. $\alpha=0.05$ was taken to indicate statistical significance.

\section{Results}

Expression of FZD2 differed across tissue types. Both overexpression and downregulation of FZD2 have been observed in human cancers relative to expression in normal samples. In
Table III. Expression of FZD2 in the SACC samples with and without metastasis and recurrence.

\begin{tabular}{lccccc}
\hline $\begin{array}{l}\text { Metastasis and } \\
\text { recurrence }\end{array}$ & $\mathrm{n}$ & Negative & Positive & $\begin{array}{c}\text { Strong } \\
\text { positive }\end{array}$ & P-value \\
\hline Yes & 19 & 13 & 5 & 1 & 0.032 \\
No & 21 & 8 & 7 & 6 & \\
\hline
\end{tabular}

Rank-sum test, $\mathrm{Z}=-2.295 ; \mathrm{P}<0.05$. SACC, salivary adenoid cystic carcinoma.

this study, we first explored the expression of FZD2 in different human cancers from the Oncomine database. According to this publicly available database, FZD2 is primarily overexpressed in colorectal, head and neck, sarcoma, lymphoma, leukemia, bladder, breast and brain cancers (Fig. 1A-D), and is downregulated in lung cancer and myeloma (Fig. 1A and E). Gene expression data for these studies are available from the Oncomine database as are the studies describing these results (http://www.oncomine.org). We also conducted an unbiased bioinformatic analysis of the gene-expression profiles of 1,609 patients with breast cancer, 1,436 patients with ovarian cancer and 1,432 patients with lung cancer using Kaplan-Meier (KM) plotter, which is a meta-analysis-based biomarker assessment tool. This analysis tool utilizes Affymetrix geneexpression profiling data and includes multiple probe sets for most genes (21-23). As displayed in Fig. 2A-C, higher expressions of FZD2 (the best cut-off was auto-selected) were associated with poor prognosis and shorter relapse-free survival among breast cancer $\left(\mathrm{P}=3.7 \times 10^{-3}\right.$, Fig. $\left.2 \mathrm{~A}\right)$ and ovarian cancer patients $\left(\mathrm{P}=4.6 \times 10^{-4}\right.$, Fig. $\left.2 \mathrm{~B}\right)$ but better prognosis and longer survival $\left(\mathrm{P}=1.3 \times 10^{-6}\right.$, Fig. $\left.2 \mathrm{C}\right)$ among lung cancer patients. In this study, we investigated the expression of FZD2 in SACCs using immunohistochemistry. As shown in Table III and Fig. 2D-F, the expression of FZD2 was downregulated in the SACC samples with metastasis and recurrence when compared to the samples without metastasis $(\mathrm{P}<0.05)$. These results imply that FZD2 may have a negative effect on the progression of adenoid cystic carcinoma.

FZD2 inhibits cell proliferation in vitro. Our real-time PCR results (Fig. 3A) revealed that FZD2 was reduced in the ACC-LM highly metastatic adenoid cystic carcinoma cell line compared to the minimally metastatic ACC- 83 cell line, which suggests that FZD2 may act as an inhibitor of the metastasis of adenoid cystic carcinomas. To investigate the effects of FZD2 on the proliferation of cancer cells, siRNA-mediated knockdown of ACC-83 was employed in ACC-83 cells. As shown by the real-time PCR (Fig. 3B), three siRNAs targeting FZD2 (siRNA-931) efficiently reduced FZD2 expression in the cells compared to the negative contros (NCs), but siRNA-931 has the highest efficiency in mRNA level and protein level (Fig. 3C). So we used siRNA-931 for further study. Knockdown of FZD2 did not increase the cell proliferation in the short term by CCK8 assay (data not shown) but it significantly promoted growth of the ACC- 83 cells in the long term ( 2 weeks) as measured by colony formation assay (Fig. 3D; $\mathrm{P}<0.01$ ). To further confirm 
A

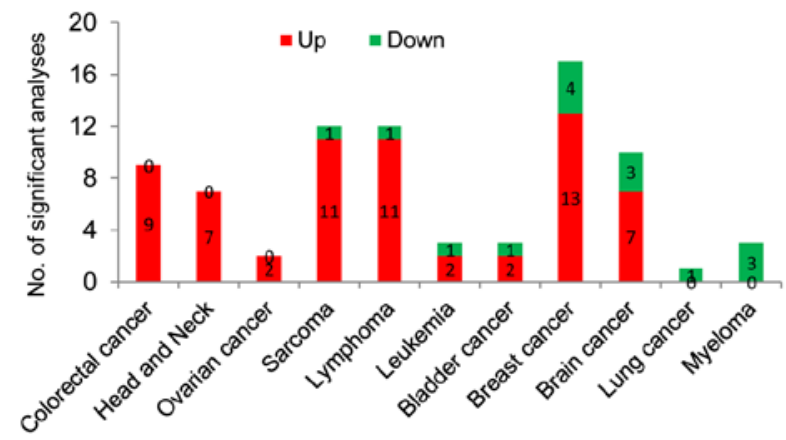

B

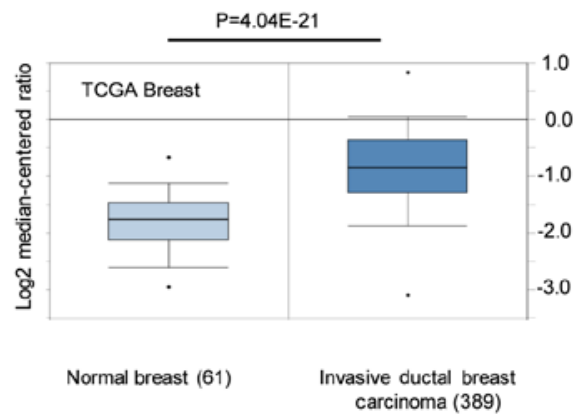

C

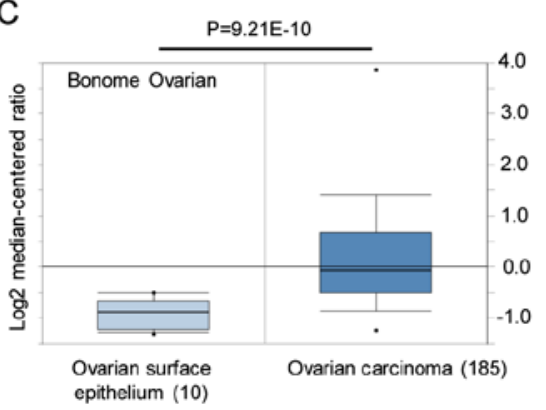

D

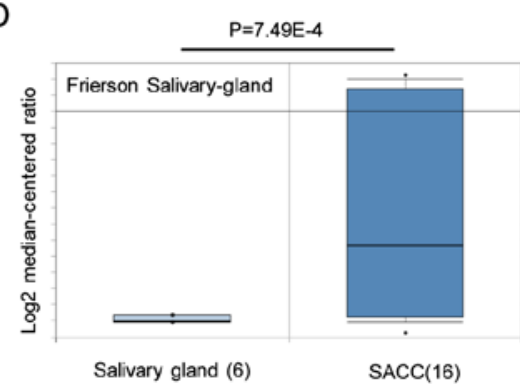

$\mathrm{E}$

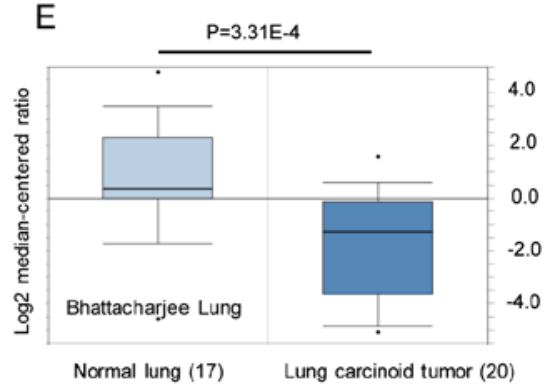

Figure 1. The deregulation of FZD2 in different cancers from the Oncomine database. (A) The number of analyses of the different types of cancers in which FZD2 was found to be upregulated (red) or downregulated (green). The threshold P-value was set to 0.01 and the fold-change was set to 1.5. (B-E) Representative images of the upregulation of FZD2 in the specified breast (B) and ovarian cancer (C), SACC datasets (D) and the downregulation in a lung cancer dataset (E).

A

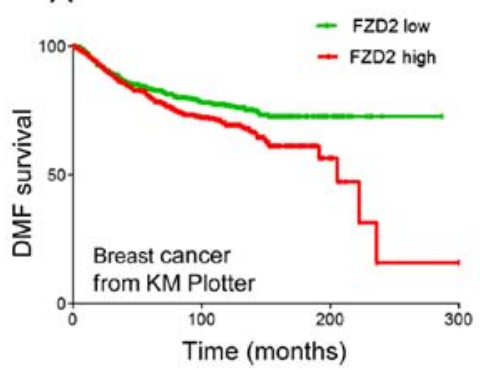

D

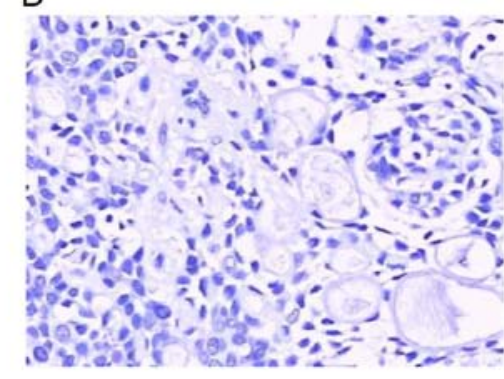

B

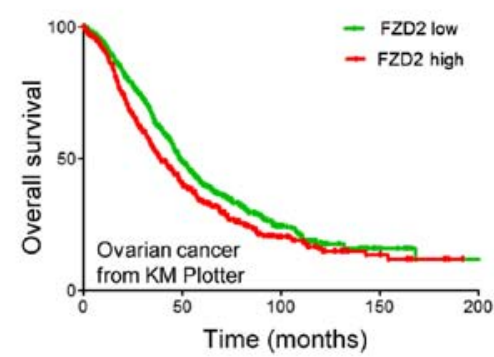

$E$

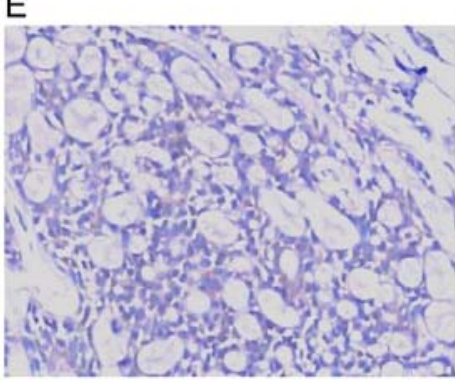

C

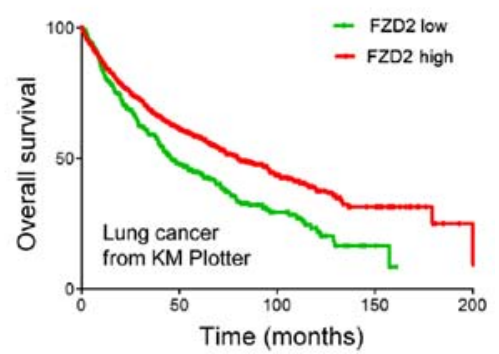

$\mathrm{F}$

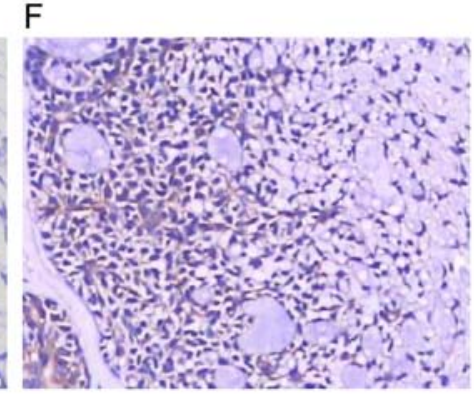

Figure 2. FZD2 is downregulated in salivary adenoid cystic carcinomas. (A-C) Survival of patients with breast (A), ovarian (B) or lung cancer (C) according to the different expression levels of FZD2 as calculated with KM plotter based on publicly available microarray data. (D and E) Representative images of the expressions of FZD2 detected in adenoid cystic carcinomas, strong positive (D), positive (E) and negative (F) (original magnification, $\mathrm{x} 400$ ).

the results of this loss-of-function study, the ACC-83 cells were transfected with an plasmid carrying the correct coding sequence of the intracellular cytoplasmic domain of FZD2. Overexpression of FZD2 in ACC-83 cells (Fig. 4A) inhibited cell proliferation over the short term as detected by the CCK8 assay (Fig. $4 \mathrm{~B} ; \mathrm{P}<0.05$ at days 3 and 4 , and $\mathrm{P}<0.01$ at day 5 ) and colony formation assay (Fig. $4 \mathrm{C} ; \mathrm{P}<0.05$ ). Collectively, these data indicate that the overexpression of FZD2 restrained SACC proliferation in vitro, which supports the notion that FZD2 is a tumor suppressor gene in SACCs.

FZD2 inhibits cell migration in vitro. Next, we examined the role of FZD2 in SACC migration. As shown in Fig. 5, knockdown of FZD2 in ACC-83 cells significantly increased 


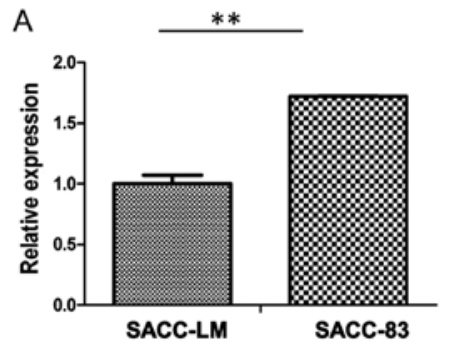

B

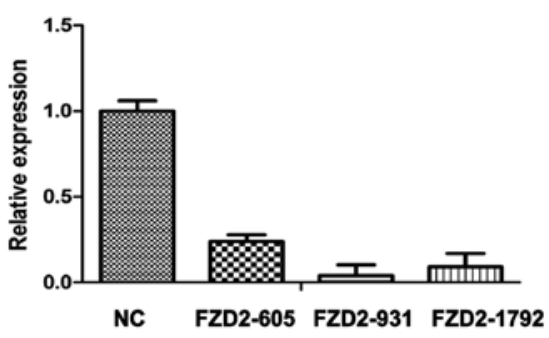

C

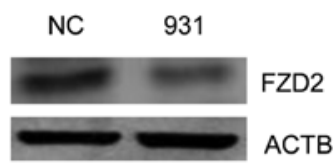

D
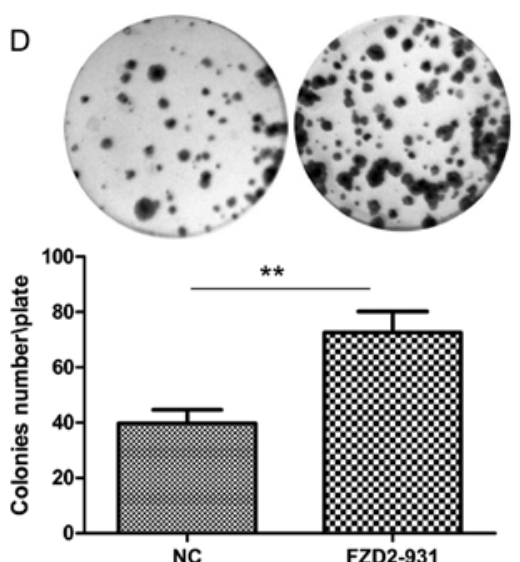

NC

Figure 3. The knockdown of FZD2 promotes the proliferation of SACC-83 cells. (A) The expressions of FZD2 in SACC-LM and SACC-83 cells were detected by real-time PCR. (B and C) Forty-eight hours after siRNA transfection, the expression of FZD2 in SACC-83 cells was measured by real-time PCR (B) and western blotting (C). (D) After siRNA transfection, the proliferation of SACC- 83 cells was increased as measured by colonies formation assay $(\mathrm{P}<0.01, \mathrm{n}=3$ ).

A

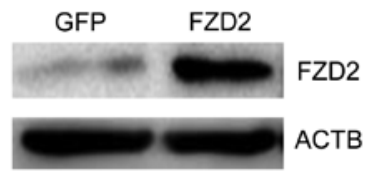

B

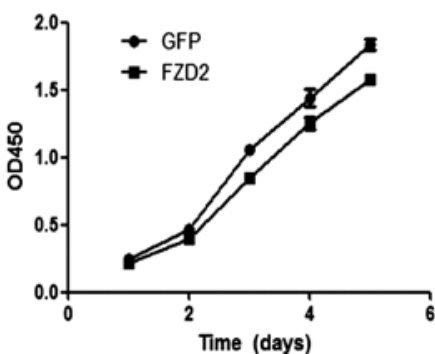

C
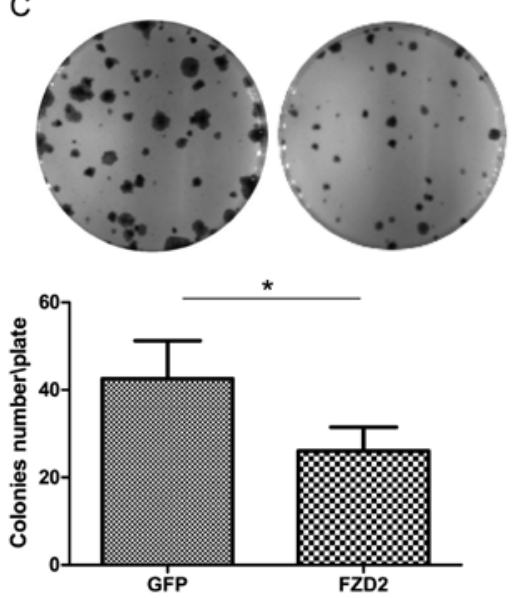

Figure 4. Overexpression of FZD2 inhibits the proliferation of SACC-83 cells. (A) Western blot analysis of the overexpression of FZD2 in SACC-83 cells transfected with plasmid. (B and C) After plasmid transfection, the proliferation of SACC-83 cells was detected with CCK-8 reagent; [(B) P<0.05 at days 3 and 4 , and $\mathrm{P}<0.01$ at day 5] and colonies formation assay $[(\mathrm{C}) \mathrm{P}<0.01, \mathrm{n}=3]$.

cell migration (Fig. 5A and $\mathrm{B} ; \mathrm{P}<0.05, \mathrm{n}=3$ ). A recent study showed that FZD2 can drive epithelial-mesenchymal transition (EMT) and cell migration through a previously unrecognized, non-canonical pathway that includes Fyn and Stat3 (24). To explore the potential mechanism of FZD2, the expression of the EMT-related genes was measured by real-time PCR after transfection of FZD2 siRNA. Compared with control, the expression of PAI-1 was downregulated after FZD2 knockdown, while the other genes like E-cadherin, vimentin and MET were not changed (Fig. 5C) in ACC-83 cells. In contrast, cell motility was decreased when FZD2 was overexpressed in ACC- 83 cells and as indicated by the results from the Transwell assay (Fig. 6A and B; P<0.001, n=3). These results demonstrate that FZD2 is a tumor suppressor gene in SACC that may inhibit the migration of ACC-83 cells.

\section{Discussion}

The Wnt signaling pathway plays multiple roles in biological development and tumorigenesis. When Wnt combines with a transmembrane protein (FZD) receptor of the Frizzled family on the cytoplasmic membrane, three intracellular signaling paths are activated $(25,26)$. In Wnt signaling activation, Wnt proteins can combine with FZD receptors and receptor complexes S(FZD/LRP) consisting of low-density lipoprotein receptor-associated proteins on the cell surface [LDL receptor 
A

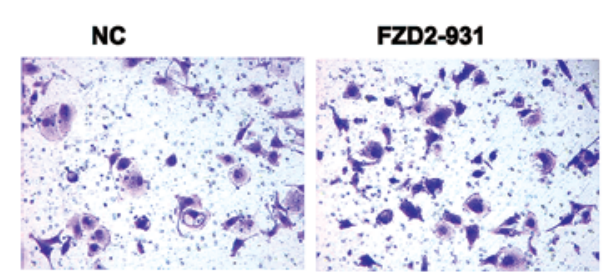

B

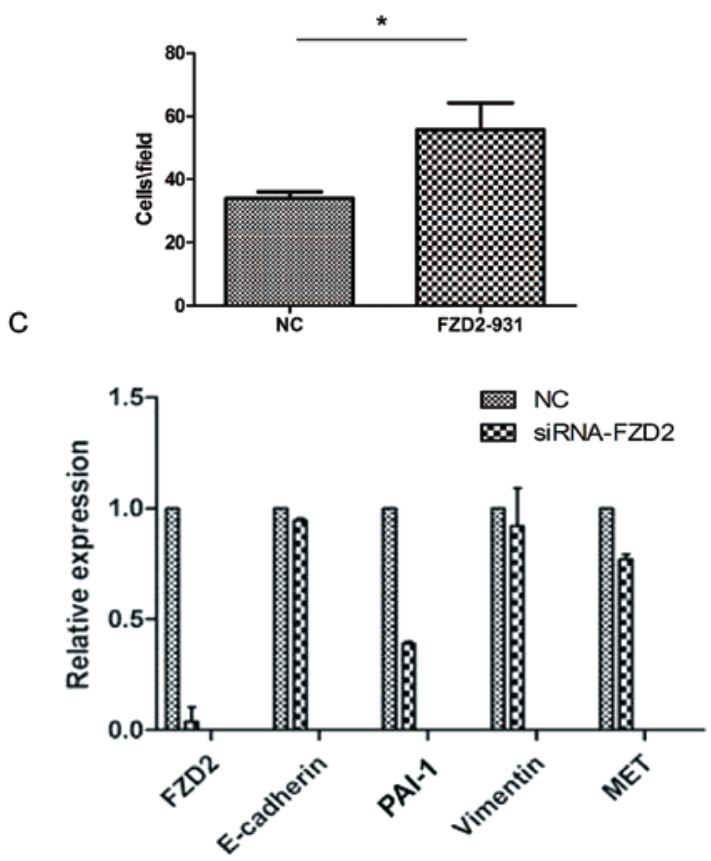

Figure 5. Knockdown of FZD2 increases the migration ability of SACC-83 cells. (A) Representative images of the Transwell assay after siRNA transfection. (B) The number of cells that migrated through the filters, which are indicative of the migratory abilities of SACC- 83 cells. The cell counts are presented as the mean values per field from at least five randomly selected low-powered fields (magnification, x200) from three independent experiments (error bars, means $\pm \mathrm{SD}$ ). $\mathrm{P}<0.05$ when compared to the controls (NC) (C) The expression of EMT related genes was measured by real-time PCR $48 \mathrm{~h}$ after FZD2 siRNA transfection.

protein (LRP)] to block $\beta$-catenin via phosphorylation and ubiquitin-mediated degradation, which produces large amounts of free $\beta$-catenin that accumulate in the cytoplasm and are then moved into the nucleus to activate the transcription and expression tumorigenesis and metastasis of target genes (27). The primary effects of Wnt-5a or Wnt-11 binding to FZD receptors are mediated by $\mathrm{Ca}^{2+}$, which activates the $\mathrm{Ca}^{2+}$ trimeric $\mathrm{G}$ protein, which in turn affects calmodulin protein-dependent kinase (CaMKII) and protein kinase $\mathrm{C}$ (PKC) to induce $\mathrm{T}$ cell nuclear factor excitation and ultimately the expression of target genes. $\mathrm{Ca}^{2+}$ channels are principally activated by low-conversion type Wnt proteins such as Wnt5a. This pathway is involved in gastrulation and also inhibits the $\beta$-catenin pathway (28). The Fizzled protein that is coded by FZD2 is one of the receptor proteins in the Wnt signaling pathway and is strongly expressed in fetal kidney, craniofacial and lung tissues and in cancers such as gastric, colon and ovarian carcinomas. Königshoff et al provided evidence that FZD2 may contribute to congenital pulmonary fibrosis based on biological analysis and qPCR identification (29). Prakash et al reported that FZD2 is involved in gastrointes-
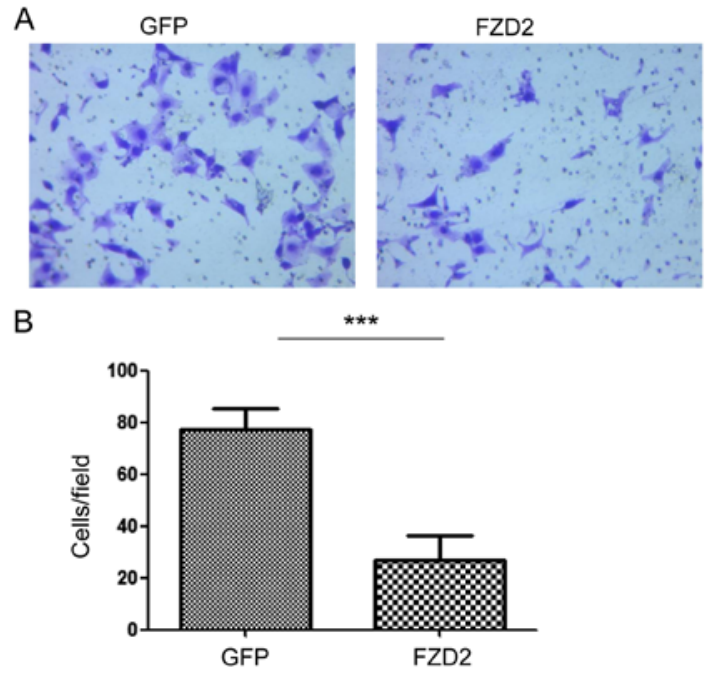

Figure 6. Overexpression of FZD2 decreases the migration ability of SACC-83 cells. (A) Representative images of the Transwell assay after plasmid transfection. (B) The number of cells that migrated through the filters were counted. The cell counts are presented as the mean values per field from at least five randomly selected low-powered fields (magnification, $\mathrm{x} 200$ ) from three independent experiments (error bars, means $\pm \mathrm{SD}$ ). $\mathrm{P}<0.001$ compared to the controls (GFP).

tinal stromal tumors in children and adolescents since FZD2 is upregulated in the younger group (30). Li et al found that the FZD2 receptor protein molecule can associate with the Wnt3a stimulated by Ror2, and this association enables the classical Wnt signaling pathway to positively regulate lung cancer (31). Kirikoshi et al utilized cDNA-PCR analysis and identification to suggest that FZD2 and FZD9 play vital roles in the occurrence of gastric cancer (32). Many studies have implied that FZD2 is closely related to the occurrence and development of numerous tumors.

Our qRT-PCR data demonstrated reduced expression of FZD2 in SACC-LM cells compared to SACC-83 cells, which is presumably related to the biological behavior of SACCs. Through immunohistochemical analyses of the protein encoded by FZD2, we found greater levels of FZD2 expression in the non-metastatic adenoid cystic carcinoma specimens than in the highly metastatic samples, which suggests that FZD2 may act as a tumor suppressor. Further investigations are warranted to clarify the increases in cell growth and migration in the SACC cells following the silencing of FZD2 and the declines following the overexpression of FZD2. Based on the above results, we speculate FZD2 is suppressive in SACCs, and the results of the present study suggest that FZD2 may have potential therapeutic applications in the treatment of SACC patients by inhibition of cell growth and metastasis.

In this study, we examined some target genes of Wnt signaling pathway in SACC cells following the study by Gujral et al (24) and found that PAI-1 was downregulated by silenced FZD2, but there were no significant changes in the expression of E-cadherin, vimentin and MET. Stefansson and Lawrence reported that PAI-1 could inhibit VSMC migration (33), while Degryse et al reported that PAI-1 could promote VSMC migration (34). The experiments carried out by Garg et al indicated PAI-1 and VN regulate each other's functions and may play key roles in VSMC migration and 
intimal hyperplasia (35). Wu et al provided evidence from wildtype mice that PAI-1 successfully inhibited the proliferation of SMCs (36). Brandal et al showed that PAI-1 inhibited the proliferation of both SMCs and ECs in proliferation assays (37). Our study uncovered that the role of tumor suppression of FZD2 in SACC may be mediated by PAI-1.

\section{Acknowledgements}

This study was supported by the National Natural Sciences Foundation of China (no. 81172583), the Natural Sciences Foundation of Fujian (project nos. 2010J01157 and 2011J01167), and the Key Project of Science and Technology Foundation of Fujian Province of China (no. 2011Y0025). The authors thank Changgong Li for providing FZD2 plasmid.

\section{References}

1. Matsuba HM, Simpson JR, Mauney M and Thawley SE: Adenoid cystic salivary gland carcinoma: A clinicopathologic correlation. Head Neck Surg 8: 200-204, 1986.

2. Rapidis AD, Givalos N, Gakiopoulou H, Faratzis G, Stavrianos SD, Vilos GA, Douzinas EE and Patsouris E: Adenoid cystic carcinoma of the head and neck. Clinicopathological analysis of 23 patients and review of the literature. Oral Oncol 41: 328-335, 2005.

3. Ampil FL and Misra RP: Factors influencing survival of patients with adenoid cystic carcinoma of the salivary glands. J Oral Maxillofac Surg 45: 1005-1010, 1987.

4. Shimoda M, Sugiura T, Imajyo I, Ishii K, Chigita S, Seki K, Kobayashi Y and Shirasuna K: The T-box transcription factor Brachyury regulates epithelial-mesenchymal transition in association with cancer stem-like cells in adenoid cystic carcinoma cells. BMC Cancer 12: 377, 2012.

5. Simonetti M, Agarwal N, Stösser S, Bali KK, Karaulanov E, Kamble R, Pospisilova B, Kurejova M, Birchmeier W, Niehrs C, et al: Wnt-Fzd signaling sensitizes peripheral sensory neurons via distinct noncanonical pathways. Neuron 83: 104-121, 2014.

6. Agarwal JP, Jain S, Gupta T, Tiwari M, Laskar SG, Dinshaw KA, Chaturvedi P, D'cruz AK and Shrivastava SK: Intraoral adenoid cystic carcinoma: Prognostic factors and outcome. Oral Oncol 44: 986-993, 2008.

7. Reya $\mathrm{T}$ and Clevers $\mathrm{H}$ : Wnt signalling in stem cells and cancer. Nature 434: 843-850, 2005.

8. Zheng D, Decker KF, Zhou T, Chen J, Qi Z, Jacobs K, Weilbaecher KN, Corey E, Long F and Jia L: Role of WNT7Binduced noncanonical pathway in advanced prostate cancer. Mol Cancer Res 11: 482-493, 2013.

9. García Campelo MR, Alonso Curbera G, Aparicio Gallego G, Grande Pulido E and Antón Aparicio LM: Stem cell and lung cancer development: Blaming the Wnt, Hh and Notch signalling pathway. Clin Transl Oncol 13: 77-83, 2011.

10. Pez F, Lopez A, Kim M, Wands JR, Caron de Fromentel C and Merle P: Wnt signaling and hepatocarcinogenesis: Molecular targets for the development of innovative anticancer drugs. J Hepatol 59: 1107-1117, 2013.

11. Dijksterhuis JP, Petersen J and Schulte G: WNT/Frizzled signalling: receptor-ligand selectivity with focus on FZD-G protein signalling and its physiological relevance: IUPHAR Review 3. Br J Pharmacol 171: 1195-1209, 2014.

12. Wang $\mathrm{Y}$ and Zheng T: Screening of hub genes and pathways in colorectal cancer with microarray technology. Pathol Oncol Res 20: 611-618, 2014.

13. Milovanovic T, Planutis K, Nguyen A, Marsh JL, Lin F, Hope C and Holcombe RF: Expression of Wnt genes and frizzled 1 and 2 receptors in normal breast epithelium and infiltrating breast carcinoma. Int J Oncol 25: 1337-1342, 2004.

14. Goksel G, Bilir A, Uslu R, Akbulut H, Guven U and Oktem G: WNT1 gene expression alters in heterogeneous population of prostate cancer cells; decreased expression pattern observed in $\mathrm{CD} 133^{+} / \mathrm{CD} 44^{+}$prostate cancer stem cell spheroids. J BUON 19 207-214, 2014

15. Ulivieri A, Lavra L, Dominici R, Giacomelli L, Brunetti E, Sciacca L, Trovato M, Barresi G, Foukakis T, Jia-Jing L, et al: Frizzled-1 is down-regulated in follicular thyroid tumours and modulates growth and invasiveness. J Pathol 215: 87-96, 2008.
16. Livak KJ and Schmittgen TD: Analysis of relative gene expression data using real-time quantitative PCR and the $2^{-\Delta \Delta C_{\mathrm{T}}}$ method. Methods 25: 402-408, 2001.

17. Su BH, Qu J, Song M, Huang XY, Hu XM, Xie J, Zhao Y, Ding LC, She L, Chen J, et al: NOTCH1 signaling contributes to cell growth, anti-apoptosis and metastasis in salivary adenoid cystic carcinoma. Oncotarget 5: 6885-6895, 2014.

18. Jinek $M$ and Doudna JA: A three-dimensional view of the molecular machinery of RNA interference. Nature 457: 405-412, 2009.

19. Snead NM and Rossi JJ: RNA interference trigger variants: Getting the most out of RNA for RNA interference-based therapeutics. Nucleic Acid Ther 22: 139-146, 2012.

20. Kanasty RL, Whitehead KA, Vegas AJ and Anderson DG: Action and reaction: The biological response to siRNA and its delivery vehicles. Mol Ther 20: 513-524, 2012.

21. Győrffy B, Surowiak P, Budczies J and Lánczky A: Online survival analysis software to assess the prognostic value of biomarkers using transcriptomic data in non-small-cell lung cancer. PLoS One 8: e82241, 2013.

22. Gyorffy B, Lánczky A and Szállási Z: Implementing an online tool for genome-wide validation of survival-associated biomarkers in ovarian-cancer using microarray data from 1287 patients. Endocr Relat Cancer 19: 197-208, 2012.

23. Sriuranpong V, Borges MW, Ravi RK, Arnold DR, Nelkin BD, Baylin SB and Ball DW: Notch signaling induces cell cycle arrest in small cell lung cancer cells. Cancer Res 61: 3200-3205, 2001.

24. Gujral TS, Chan M, Peshkin L, Sorger PK, Kirschner MW and MacBeath G: A noncanonical Frizzled2 pathway regulates epithelial-mesenchymal transition and metastasis. Cell 159: 844-856, 2014.

25. MacDonald BT, Hien A, Zhang X, Iranloye O, Virshup DM, Waterman ML and He X: Disulfide bond requirements for active Wnt ligands. J Biol Chem 289: 18122-18136, 2014.

26. Kahn M: Can we safely target the WNT pathway? Nat Rev Drug Discov 13: 513-532, 2014

27. Daniels DL and Weis WI: Beta-catenin directly displaces Groucho/TLE repressors from Tcf/Lef in Wnt-mediated transcription activation. Nat Struct Mol Biol 12: 364-371, 2005.

28. van de Schans VA, Smits JF and Blankesteijn WM: The Wnt/ frizzled pathway in cardiovascular development and disease: Friend or foe? Eur J Pharmacol 585: 338-345, 2008.

29. Königshoff M, Balsara N, Pfaff EM, Kramer M, Chrobak I, Seeger $\mathrm{W}$ and Eickelberg O: Functional Wnt signaling is increased in idiopathic pulmonary fibrosis. PLoS One 3: e2142, 2008.

30. Prakash S, Sarran L, Socci N, DeMatteo RP, Eisenstat J, Greco AM, Maki RG, Wexler LH, LaQuaglia MP, Besmer P, et al: Gastrointestinal stromal tumors in children and young adults: A clinicopathologic, molecular, and genomic study of 15 cases and review of the literature. J Pediatr Hematol Oncol 27: 179-187, 2005.

31. Li C, Chen H, Hu L, Xing Y, Sasaki T, Villosis MF, Li J, Nishita M, Minami Y and Minoo P: Ror2 modulates the canonical Wnt signaling in lung epithelial cells through cooperation with Fzd2. BMC Mol Biol 9: 11-22, 2008.

32. Kirikoshi H, Sekihara $\mathrm{H}$ and Katoh M: Expression profiles of 10 members of Frizzled gene family in human gastric cancer. Int $\mathbf{J}$ Oncol 19: 767-771, 2001.

33. Stefansson S and Lawrence DA: The serpin PAI-1 inhibits cell migration by blocking integrin alpha $\mathrm{V}$ beta 3 binding to vitronectin. Nature 383: 441-443, 1996.

34. Degryse B, Neels JG, Czekay RP, Aertgeerts K, Kamikubo Y and Loskutoff DJ: The low density lipoprotein receptor-related protein is a motogenic receptor for plasminogen activator inhibitor-1. J Biol Chem 279: 22595-22604, 2004.

35. Garg N, Goyal N, Strawn TL, Wu J, Mann KM, Lawrence DA and Fay WP: Plasminogen activator inhibitor-1 and vitronectin expression level and stoichiometry regulate vascular smooth muscle cell migration through physiological collagen matrices. J Thromb Haemost 8: 1847-1854, 2010.

36. Wu J, Peng L, McMahon GA, Lawrence DA and Fay WP: Recombinant plasminogen activator inhibitor-1 inhibits intimal hyperplasia. Arterioscler Thromb Vasc Biol 29: 1565-1570, 2009.

37. Brandal S, Blake CM, Sullenger BA and Fortenberry YM: Effects of plasminogen activator inhibitor-1-specific RNA aptamers on cell adhesion, motility, and tube formation. Nucleic Acid Ther 21: 373-381, 2011. 